\title{
UNIFORM DISTRIBUTION OF SEQUENCES OF INTEGERS
}

\author{
BY \\ IVAN NIVEN(1)
}

1. Introduction. Consider an infinite sequence $A$ of integers, $A=\left\{a_{i}\right\}$. For any integers $j$ and $m \geqq 2$ define $A(n, j, m)$ as the number of terms among $a_{1}, a_{2}, a_{3}, \cdots, a_{n}$ that satisfy $a_{i} \equiv j(\bmod m)$. We say that the sequence $A$ is uniformly distributed modulo $m$ in case

$$
\lim _{n \rightarrow \infty} \frac{1}{n} A(n, j, m)=\frac{1}{m} \quad \text { for } j=1,2,3, \cdots, m .
$$

For example any arithmetic progression $\{a n+b ; n=1,2,3, \ldots\}$ is uniformly distributed modulo $m$ if and only if g.c.d. $(a, m)=1$. Further we say that the sequence $A$ is uniformly distributed in case $A$ is uniformly distributed modulo $m$ for every integer $m \geqq 2$. The case $m=1$ is omitted because $A(n, j, 1)=n$, and so (1.1) holds for every sequence $A$ in case $m=1$. Furthermore the language "uniformly distributed modulo 1 " has a well-established meaning for a sequence of real numbers $\left\{\alpha_{n}\right\} ;$ cf. [1] or [2, p. 72].

These definitions apply to any sequence of integers. Our interest will be primarily in sequences of positive integers satisfying $a_{i}<a_{i+1}$, at least for $i$ sufficiently large. For such sequences, the definitions can be given in an alternative form. First define $Q(n, j, m)$ as the number of terms $a_{i}$ of the sequence $A$ that satisfy the conditions $a_{i} \leqq n$ and $a_{i} \equiv j(\bmod m)$. Also define $A(n)$ as the number of terms $a_{i}$ of $A$ satisfying $a_{i} \leqq n$, so that $A(n)=Q(n, j, 1)$ for any $j$. Then the sequence $A$ is uniformly distributed modulo $m$ in case

$$
\lim _{n \rightarrow \infty} \frac{a(n, j, m)}{A(n)}=\frac{1}{m} \quad \text { for } j=1,2,3, \cdots, m .
$$

Although we shall occasionally use (1.2) when it is applicable, it should be understood that the more general (1.1) will serve as the definition of uniform distribution. For example (1.1) can be applied to the sequence of negative integers $-1,-2,-3, \cdots$, whereas (1.2) cannot.

The purpose of this paper is to investigate some properties of uniformly distributed sequences. In $\$ 2$ we discuss the circumstances under which the complementary sequence $\bar{A}$ of a sequence $A$ of positive integers is uniformly

Presented to the Society, September 2, 1960; received by the editors May 13, 1960.

(1) Supported in part by the Office of Naval Research. 
distributed, if $A$ is. In $\S 3$ we prove that for any irrational number $\theta$ the sequence $[\theta],[2 \theta],[3 \theta], \cdots$ is uniformly distributed, where by $[n \theta]$ is meant the customary value, the greatest integer not exceeding $n \theta$. This is proved as a consequence of the theorem of $\mathrm{H}$. Weyl that the fractional parts, i.e., $n \theta-[n \theta]$, are uniformly distributed in the unit interval. We also show that the Weyl theorem is a consequence of, and so equivalent to, the result that the sequence $\{[n \theta] ; n=1,2,3, \cdots\}$ is uniformly distributed. Some further questions of a similar nature are also discussed in $\$ 3$, and also the question of the cardinal number of uniformly and nonuniformly distributed sequences. In $\$ 4$ it is proved that the only polynomials $f(x)$ with integer coefficients such that $f(1), f(2), f(3), \cdots$ are uniformly distributed are the polynomials $\pm x+c$. Some questions of the relationship of uniform distribution modulo $m$ and modulo $k$ are treated in $\$ 5$. In the final $\S 6$, a property involving exponential sums is discussed.

An immediate consequence of the definitions is that a sequence $A=\left\{a_{i} ; i \geqq 1\right\}$ is uniformly distributed modulo $m$ if and only if the truncated sequence $A_{r}=\left\{a_{i} ; i \geqq r\right\}$ is uniformly distributed $\bmod m$ for any fixed positive integer $r$. Thus if $A$ is uniformly distributed, so is $A_{r}$ and conversely.

Similarly, $A$ is uniformly distributed modulo $m$ if and only if the sequence $A+c=\left\{a_{i}+c ; i \geqq 1\right\}$ is uniformly distributed modulo $m$, where $c$ is any integer.

2. Complementary sequences. Consider any strictly increasing sequence $A$ of positive integers whose complement $\bar{A}$ is an infinite sequence. If $A$ is uniformly distributed, must $\bar{A}$ also be? If $A$ is uniformly distributed modulo $m$, must $\bar{A}$ also be? In both cases the answer is no. For example let $A$ be the sequence of composite integers $4,6,8,9,10, \cdots$. For every $m \geqq 2$, this particular set $A$ is uniformly distributed modulo $m$. However, the complementary sequence comprises 1 and the prime numbers, thus $1,2,3,5,7, \cdots$, and this sequence is not uniformly distributed modulo $m$ for any $m \geqq 2$.

The fact that the composite integers are uniformly distributed modulo $m$ for every $m$ can be regarded as a special case of a more general proposition: If a sequence $A$ consists of all positive integers except for a set of natural density zero, then $A$ is uniformly distributed modulo $m$ for every $m \geqq 2$. (A sequence $B$ of increasing positive integers has natural density zero in case $B(n) / n \rightarrow 0$ as $n \rightarrow \infty$. More generally the asymptotic density of $B$ is $\lim \inf B(n) / n$, and $B$ has natural density $\lim B(n) / n$ if this limit exists.)

However, $\bar{A}$ inherits the uniform distribution property from $A$ in case $\bar{A}$ has positive density.

TheOREM 2.1. Let $A$ be a strictly increasing sequence of positive integers whose complement $\bar{A}$ has positive asymptotic density. Then if $A$ is uniformly distributed modulo $m$, so is $\bar{A}$. Also if $A$ is uniformly distributed, so is $\bar{A}$.

Proof. It suffices to prove the first of the two assertions. By $\bar{a}(n, j, m)$ is 
meant the number of integers $\vec{a}$ in the sequence $\bar{A}$ that satisfy the conditions $\bar{a} \leqq n$ and $\bar{a} \equiv j(\bmod m)$. Then we have

$$
a(n, j, m)+\bar{a}(n ; j, m)=\frac{n}{m}+\alpha, \quad|\alpha|<1,
$$

or

$$
\frac{a(n, j, m)}{A(n)} \cdot \frac{A(n)}{n}+\frac{\bar{a}(n, j, m)}{\bar{A}(n)} \cdot \frac{\bar{A}(n)}{\bar{A}(n)}=\frac{1}{m}+\frac{\alpha}{n},
$$

where $\bar{A}(n)$ denotes the number of integers $\bar{a}$ of $\bar{A}$ satisfying $\bar{a} \leqq n$. Now $A(n)+\bar{A}(n)=n$, so we can replace the fraction $A(n) / n$ by $1-\bar{A}(n) / n$ to get

$$
\frac{\bar{A}(n)}{n}\left\{\frac{\bar{\alpha}(n, j, m)}{\bar{A}(n)}-\frac{a(n, j, m)}{A(n)}\right\}=\frac{1}{m}-\frac{a(n, j, m)}{n}+\frac{\alpha}{n} .
$$

As $n \rightarrow \infty$, the right side tends to zero, by (1.2) since we are assuming that $A$ is uniformly distributed modulo $m$. Since $\bar{A}$ is assumed to have positive asymptotic density, we know that there is a positive $\delta$ so that $\bar{A}(n) / n>\delta$ for all $n$ sufficiently large, and so from (2.1) we conclude that for every $j$

$$
\lim _{n \rightarrow \infty} \frac{\bar{a}(n, j, m)}{\bar{A}(n)}=\lim _{n \rightarrow \infty} \frac{a(n, j, m)}{A(n)}=\frac{1}{m} .
$$

THEOREM 2.2. If a sequence $A$ of positive integers is uniformly distributed and contains an infinite arithmetic progression, then $A$ has density 1.

Proof. Let $A$ contain $\{b k+c\}$ for all $k$ sufficiently large. Then $a(n, c, b)$ as in (1.2) equals $n / b+o(n)$. Hence we have

$$
\lim _{n \rightarrow \infty} \frac{a(n, c, b)}{A(n)}=\frac{1}{b}=\lim \frac{n / b+o(n)}{A(n)}=\frac{n}{b A(n)}
$$

and so $\lim A(n) / n=1$.

3. Multiples of an irrational number.

THEOREM 3.1. For any real number $\theta$ let $S(\theta)$ denote the sequence of integers

$$
[\theta],[2 \theta],[3 \theta],[4 \theta], \cdots,[n \theta], \cdots \text {. }
$$

$S(\theta)$ is uniformly distributed if and only if $\theta$ is irrational or $\theta=1 / n$ for some nonzero integer $n$.

Proof. For $\theta$ irrational, this result is a consequence of the result of $\mathrm{H}$. Weyl that the sequence of real numbers

$$
\theta-[\theta], 2 \theta-[2 \theta], 3 \theta-[3 \theta], 4 \theta-[4 \theta], \cdots
$$


is uniformly distributed modulo 1 ; $\mathrm{cf}$. [1], or [2, pp. 72-81]. We apply the Weyl theorem to the irrational number $\theta / m$ for any integer $m \geqq 2$. Thus we conclude that the sequence of real numbers $n \theta / m-[n \theta / m]$ for $n=1,2,3, \ldots$ is uniformly distributed modulo 1 , in fact uniformly distributed in the unit interval. Multiplying the terms of this sequence by $m$, we conclude that the sequence $S_{1}$ of real numbers $n \theta-m[n \theta / m]$ for $n=1,2,3, \ldots$ is uniformly distributed in the limit sense over the interval $0 \leqq x \leqq m$ on the real line. Hence if we take the integer parts of the members of the sequence $S_{1}$,

$$
[n \theta-m[n \theta / m]]=[n \theta]-m[n \theta / m], \quad n=1,2,3, \cdots,
$$

we see that the terms of this sequence are uniformly distributed modulo $m$ because they are uniformly distributed among the values $0,1,2, \cdots, m-1$. Finally if the terms $m[n \theta / m]$ are removed from the sequence (3.2), the uniformity of the distribution modulo $m$ is not disturbed, and so we have proved the theorem in case $\theta$ is irrational.

In case $\theta$ is rational, we can confine our attention to positive $\theta$ since there is symmetry in the results for negative $\theta$. We write $\theta=a / b$ where $a$ and $b$ are positive integers with $(a, b)=1$. First consider the case where $a>b$. Then the integers $[k \theta]$ for $k=1,2,3, \cdots, n b$ have the property that at least $n$ of them are multiples of $a$, namely in the cases $k=b, 2 b, 3 b, \cdots, n b$. Thus in the limit, at least $1 / b$ of the members of $S(\theta)$ are congruent to zero modulo $a$. Hence $S(\theta)$ is not uniformly distributed modulo $a$.

Next consider the case where $a \leqq b$, and define $r$ as the least integer such that $r a \geqq b>(r-1) a$. Then among the integers $[k \theta]$ for $k=1,2, \cdots, n b$ $+r-1$, at least $n r$ are multiples of $a$, namely the cases $k=j b+i$ with $1 \leqq j \leqq n$ and $0 \leqq i \leqq r-1$. Thus of the $n b+r-1$ members of $S(\theta)$ under consideration, at least the fraction $n r /(n b+r-1)$ are multiples of $a$. This fraction tends to $r / b$ as $n$ tends to infinity. So if the sequence $S(\theta)$ is to be uniformly distributed modulo $a$ it is necessary that $r / b \leqq 1 / a$, that is $b \geqq r a$. Now by definition $r a \geqq b$, so we have $b=r a$. But $(a, b)=1$ so the condition becomes $a=1, b=r$.

This condition is also sufficient, for if $\theta=1 / b$ then the sequence $[n \theta]$ for $n=1,2,3, \cdots$ is readily seen to be uniformly distributed.

Theorem 3.1 can be generalized to other sequences

$$
f(\theta), f(2 \theta), f(3 \theta), f(4 \theta), \cdots .
$$

For example if $\theta$ is irrational and $f(n \theta)$ is taken to mean the least integer $\geqq n \theta$, or the nearest integer to $n \theta$, then the sequence (3.3) is uniformly distributed. These results are special cases of the following theorem, namely with $\beta=1$ and $\beta=1 / 2$ respectively.

THEOREM 3.2. Let $\beta$ be any fixed real number, let $\theta$ be irrational, and define $f(n \theta)=[n \theta+\beta]$. Then the sequence (3.3) is uniformly distributed.

Proof. If in the notation of the proof of Theorem 3.2 we add $\beta$ to each of 
the terms of $S_{1}$, we get the sequence $S_{2}$ of real numbers $n \theta+\beta-m[n \theta / m]$ for $n=1,2,3, \ldots$ whose terms are uniformly distributed over the interval $\beta \leqq x \leqq \beta+m$. Hence the integer parts of the terms of $S_{2}$ are uniformly distributed modulo $m$; likewise the sequence $\{f(n \theta)\}$.

We give one further variation on sequences of integers defined by an irrational number.

Theorem 3.3. If $\theta$ is irrational, $|\theta|<1$, then the following sequence of integers is uniformly distributed: all positive integers $a_{i}$ such that there is an integer between $\theta a_{i}$ and $\theta\left(1+a_{i}\right)$, that is such that $\left[\theta a_{i}\right]<\left[\theta\left(1+a_{i}\right)\right]$.

Proof. It suffices to prove this for $\theta$ positive. Define the integer $b_{i}$ by

$$
\theta a_{i}<b_{i}<\theta\left(1+a_{i}\right), \text { so } a_{i}<\frac{b_{i}}{\theta}<1+a_{i}, \quad a_{i}=\left[b_{i} / \theta\right] .
$$

However, we note that $b_{i}=i$, so the sequence $a_{i}$ is the same as the sequence $[i / \theta] ; i=1,2,3, \cdots$. But this sequence is uniformly distributed by Theorem 3.1 .

It may be noted that the uniform distribution of the sequence of integers $\{[n \theta] ; n=1,2,3, \cdots\}$ for every irrational $\theta$ implies the Weyl theorem that the fractional parts of $n \theta$ are uniformly distributed modulo 1 . An argument for this can be made along the following lines. For any positive integer $m$ the sequence $\{[n m \theta] ; n=1,2,3, \cdots\}$ is presumed to be uniformly distributed, and so uniformly distributed modulo $m$.

Let $j$ be any integer satisfying $0 \leqq j<m$. For any $n$ such that $[n m \theta]$ $\equiv j(\bmod m)$ we see that

$$
\begin{gathered}
{[n m \theta]-m[n \theta]=j,} \\
j<n m \theta-m[n \theta]<j+1, \\
\frac{j}{m}<n \theta-[n \theta]<\frac{j+1}{m} .
\end{gathered}
$$

Hence the fractional parts of $n \theta, n=1,2,3, \cdots$ are, in the limit, equally divided among the intervals

$$
\left(0, \frac{1}{m}\right),\left(\frac{1}{m}, \frac{2}{m}\right),\left(\frac{2}{m}, \frac{3}{m}\right), \ldots,\left(\frac{m-1}{m}, 1\right)
$$

for every positive integer $m$. As a consequence of this we see that the fractional parts of the sequence $\{n \theta\}$ are uniformly distributed modulo 1 .

We now discuss the cardinality of the set $\Sigma$ of uniformly distributed sequences of positive integers. Since by Theorem 3.1 the set $\Sigma$ contains as members all $S(\theta)$ with $\theta$ irrational, $\theta>1$, we conclude that $\Sigma$ has at least the cardinal number of the continuum. And $\Sigma$ must have in fact this cardinal 
number because the set of all sequences of positive integers has the cardinal number of the continuum.

Let us map every sequence $A=\left\{a_{k}\right\}$ of positive integers onto an infinite decimal in the binary system, with a digit 1 in the position $a_{k}$ for $k \geqq 1$, and zeros elsewhere. For example the sequence of odd integers $1,3,5,7, \ldots$ maps onto $.10101010 \ldots$. Thus we have a one to one correspondence between infinite sequences $A$ of positive integers and the real numbers $x$ satisfying $0<x \leqq 1$, with each $x$ expressed in the binary system. Now if $x$ is a normal number to base two (cf. [3] or [2, pp. 94-104]), then the corresponding sequence $A$ is uniformly distributed, but not conversely. Since almost all numbers are normal to base two, we see that in the sense of the above mapping almost all sequences of positive integers are uniformly distributed.

The nonuniformly distributed sequences also have the cardinal number of the continuum. In fact for any given $m$ the set of sequences which are not uniformly distributed modulo $m$ has the cardinal of the continuum. For if $A=\left\{a_{k}\right\}$ is any uniformly distributed sequence, then $A^{*}=\left\{m a_{k}\right\}$ is not uniformly distributed modulo $m$, and distinct sequences $A$ yield distinct sequences $A^{*}$.

\section{Integers defined by polynomials.}

THEOREM 4.1. Let $f(x)$ be any polynomial with integer coefficients. Then the sequence $\{f(n) ; n=1,2,3, \cdots\}$ is uniformly distributed if and only if $f(x)$ is of the form $\pm x+c$. Moreover if $f(x)$ is not linear, then there are infinitely many integers $m$ for which the sequence $\{f(n)\}$ is not uniformly distributed modulo $m$.

Proof. We have already noted in $\$ 1$ that in case $f(x)=a x+b$ the sequence $f(n)$ is uniformly distributed modulo $m$ if and only if the g.c.d. $(a, m)=1$. Hence the theorem follows for linear polynomials, because $(a, m)=1$ for all $m \geqq 2$ implies that $a= \pm 1$.

Turning to nonlinear polynomials, we observe that it suffices to treat polynomials with zero constant terms, because the sequences $\{f(n)\}$ and $\{f(n)-f(0)\}$ are both uniformly distributed or both not. Thus we pass from $f(x)$ to $F(x)=f(x)-f(0)$, and we first dispense with the case where $F(x)$ is a monomial, say $F(x)=a x^{k}$ with $k \geqq 2$. Then for no value of $n$ is it true that $F(n) \equiv p\left(\bmod p^{2}\right), p$ any prime, and so the sequence $\{F(n)\}$ is not uniformly distributed modulo any $p^{2}$.

If $F(x)$ is not a monomial, we can write

$$
\begin{aligned}
F(x) & =x^{j} g(x), & j \geqq 1, \\
g(x) & =\sum_{i=0}^{k} a_{i} x^{i}, & k \geqq 1, a_{0} \neq 0, a_{k} \neq 0 .
\end{aligned}
$$

Now it is well known that for any nonconstant polynomial $g(x)$ there are infinitely many primes $p$ such that $g(x) \equiv 0(\bmod p)$ is solvable $[4$, p. 82$]$. 
Choose such a prime $p>a_{0}$, and let $r$ be an integer such that $g(r) \equiv 0(\bmod p)$. Then $r \not \equiv 0(\bmod p)$ because $g(0) \equiv a_{0} \not \equiv 0(\bmod p)$. Hence $F(x) \equiv 0(\bmod p)$ has solutions $x \equiv r(\bmod p)$ and $x \equiv 0(\bmod p)$, that is

$$
F(r+n p) \equiv F(n p) \equiv 0(\bmod p) \quad \text { for } n=1,2,3, \cdots .
$$

If $S$ denotes the sequence $\{F(n)\}$, it follows that

$$
\lim \inf \frac{1}{n} S(n, o, p) \geqq \frac{2}{p},
$$

and so $S$ is not uniformly distributed for any such prime $p$.

5. Independence of moduli. If a sequence $S$ is uniformly distributed modulo $m$, and $k \mid m$, then it is clear that $S$ is uniformly distributed modulo $k$. We show that no other implication holds.

THEOREM 5.1. If a sequence is uniformly distributed $\bmod m$, then it is uniformly distributed mod every divisor of $m$. If $k$ is not a divisor of $m$ then there is a sequence that is uniformly distributed $\bmod m$ but not $\bmod k$.

Proof. If $k \nmid m$, write $v$ for the l.c.m. of $k$ and $m$. Then consider the set $S$ which is the union of the arithmetic progressions $\{n v+i ; n=1,2,3, \cdots\}$ for $i=0,1,2, \cdots, m-1$. Then $S$ is uniformly distributed modulo $m$ but not modulo $k$.

Suppose a sequence is uniformly distributed with respect to relatively prime moduli $m$ and $k$, so that $(m, k)=1$. Then must the sequence be uniformly distributed modulo $m k$ ? The answer is no, as the following example shows. Let $A$ be the sequence of all positive integers $a$ such that $a \equiv 1,2,3,6$, 7 , or $8(\bmod 12)$. Then $A$ is uniformly distributed modulo 2 and modulo 3 , but not modulo 6 .

If a sequence $A$ is not uniformly distributed, then there are infinitely many moduli $m$ for which $A$ is not uniformly distributed. For there must be some modulus $k$ for which $A$ is not uniformly distributed, and all multiples of $k$ have the same property.

However, it turns out that there may be no prime powers among the moduli for which $A$ is not uniformly distributed. We now construct a specific sequence $A$ which is not uniformly distributed in the general sense, but is uniformly distributed modulo $p^{\alpha}$ for every prime $p$ and every integer $\alpha \geqq 1$. Let $p_{r}$ denote the $r$ th prime. Given any positive integer $k$, for each prime $p_{r} \leqq k$ define $j=j(r, k)$ by the inequalities $p_{r}^{j} \leqq k<p^{j+1}$. Then $a_{k}$ will be chosen to satisfy certain conditions, the first being $a_{k}>a_{k-1}$, the second being

$$
a_{k} \equiv k\left(\bmod p_{r}^{j}\right)
$$

for every $p_{r} \leqq k$ except $p_{1}$. As to the prime $p_{1}$, we use a variation on (5.1) thus. If $k$ is of the form $6 q+3$ or $6 q+4$ we reverse the congruence conditions as follows: 


$$
\begin{array}{ll}
a_{k}=k+1\left(\bmod p_{1}^{j}\right) & \text { if } k=6 q+3, \\
a_{k}=k-1\left(\bmod p_{1}^{j}\right) & \text { if } k=6 q+4 .
\end{array}
$$

For all other values of $k$ we use (5.1) with $r=1$. Thus we have imposed a finite number of compatible congruence conditions on each $a_{k}$ with $k>1$; define $a_{1}=1$.

Then the sequence $\left\{a_{k}\right\}$ is uniformly distributed modulo $p_{r}^{\alpha}$ for all $r \geqq 1$ and all $\alpha \geqq 1$. However, if $k \equiv 0(\bmod 3)$ then $a_{k} \equiv 0(\bmod 3)$ and $a_{k} \equiv 0(\bmod 2)$. If $k \neq \equiv 0(\bmod 3)$ then $a_{k} \not \equiv 0(\bmod 3)$. Hence there are no values of $a_{k} \equiv 3(\bmod 6)$, and so the sequence $\left\{a_{k}\right\}$ is not uniformly distributed.

6. A closely related property. Say that a sequence $A=\left\{a_{k}\right\}$ has the property $P_{m}$ in case

$$
\lim _{n \rightarrow \infty} \frac{1}{n} \sum_{k=1}^{n} \exp \left(2 \pi i a_{k} / m\right)=0 .
$$

If $A$ is uniformly distributed modulo $m$, then $A$ has property $P_{m}$ because

$$
\begin{aligned}
\sum_{k=1}^{n} \exp \left(2 \pi i a_{k} / m\right) & =\sum_{j=1}^{m} A(n, j, m) \exp (2 \pi i j / m) \\
& =\sum_{j=1}^{m}\left\{\frac{n}{m}+o(n)\right\} \exp (2 \pi i j / m)=\sum_{j=1}^{m}\{o(n)\} \exp (2 \pi i j / m) \\
& =o(n) .
\end{aligned}
$$

However the converse does not hold except for $m=2$ and $m=3$. If $A$ has the property $P_{3}$ then

$$
\begin{array}{r}
0=\lim \frac{1}{n} \sum_{k=1}^{n} \exp \left(2 \pi i a_{k} / 3\right)=\lim \frac{1}{n} \sum_{j=1}^{3} A(n, j, 3) \exp (2 \pi i j / 3) \\
=\lim \frac{1}{n}\left\{A(n, 3,3)-\frac{1}{2} A(n, 1,3)-\frac{1}{2} A(n, 2,3)+\frac{i(3)^{1 / 2}}{2} A(n, 1,3)\right. \\
\left.-\frac{i(3)^{1 / 2}}{2} A(n, 2,3)\right\} .
\end{array}
$$

Taking limits of the real and imaginary parts separately, and using the fact that $A(n, 3,3)=n-A(n, 1,3)-A(n, 2,3)$ we obtain

$$
\lim \frac{1}{n}\{A(n, 1,3)-A(n, 2,3)\}=0
$$

and

$$
\lim \frac{1}{n}\{A(n, 1,3)+A(n, 2,3)\}=\frac{2}{3}
$$


so that

$$
\lim \frac{1}{n} A(n, 1,3)=\lim \frac{1}{n} A(n, 2,3)=\lim \frac{1}{n} A(n, 3,3)=\frac{1}{3},
$$

so that $A$ is uniformly distributed modulo 3 . Similarly if $A$ has the property $P_{2}$, an analogous proof shows that $A$ is uniformly distributed modulo 2 .

But $A$ having the property $P_{m}$ does not imply that $A$ is uniformly distributed modulo $m$ for any $m>3$. To see this in case $m$ is even, say $m=2 q$, we need only take the sequence $\{n q ; n=1,2,3, \ldots\}$. In case $m$ is odd, say $m=2 q+1$ with $q>1$, we can take $A$ to be the union of the three sequences

$$
\{n m\}, \quad\{q+[n \theta] m\}, \quad\{q+1+[n \theta] m\}, \quad n=1,2,3, \cdots,
$$

where $\theta=2 \cos \pi / m$.

Consider the question: If a sequence $A$ has the property $P_{m}$ for all $m \geqq 2$, then is $A$ necessarily uniformly distributed? The answer is no, as the following example shows. Define $B=\left\{b_{k}\right\}$ as the sequence defined by $b_{k}=5[k \theta]$ where $\theta=2 \tan \pi / 5>1$. Now the sequence $\left\{b_{k} / 5\right\}$ is uniformly distributed, by Theorem 3.1. Consequently the sequence $\left\{b_{k} / 5\right\}$ has the property $P_{m}$ for all $m \geqq 2$, and it follows that the sequence $B=\left\{b_{k}\right\}$ has the property $P_{m}$ for all $m \geqq 2$ except $m=5$.

Next define $C$ as the sequence $\dot{c}_{k}$ where

$$
c_{k}=5[k \alpha]+2, \quad \alpha=4 \sin \pi / 5>1 .
$$

Then by the same argument as in the case of $B$, we conclude that $C$ has the property $P_{m}$ for all $m \geqq 2$ except $m=5$. The same is also true of the sequence $D=\left\{d_{k}\right\}$ defined by

$$
d_{k}=5[k \alpha]+3, \quad \alpha=4 \sin \pi / 5>1 .
$$

Define $A$ as the sequence obtained by taking the union of $B, C$, and $D$, the terms of $A$ being enumerated in increasing size. Since $B, C$, and $D$ are disjoint, their union $A$ inherits their property $P_{m}$ for $m>2, m \neq 5$. But it turns out that $A$ has property $P_{5}$ also.

To prove this, we write $\delta(B)$ for the natural density of $B$ (cf. $\S 2$ ) so that $\delta(B)=(5 \theta)^{-1}$. Likewise we have $\delta(C)=\delta(D)=(5 \alpha)^{-1}$. Suppose that the first $n$ terms of the sequence $A$ are made up of $n_{1}$ terms from $B, n_{2}$ terms from $C$, and $n_{3}$ terms from $D$. Then using the values of the natural densities of these sets, we conclude that

$$
\begin{aligned}
& \lim _{n \rightarrow \infty} \frac{n_{1}}{n}=\frac{(5 \theta)^{-1}}{(5 \theta)^{-1}+2(5 \alpha)^{-1}}=\frac{\alpha}{2 \theta+\alpha}, \\
& \lim _{n \rightarrow \infty} \frac{n_{2}}{n}=\lim _{n \rightarrow \infty} \frac{n_{3}}{n}=\frac{(5 \alpha)^{-1}}{(5 \theta)^{-1}+2(5 \alpha)^{-1}}=\frac{\theta}{2 \theta+\alpha} .
\end{aligned}
$$


Hence we can write

$$
\begin{aligned}
\lim _{n \rightarrow \infty} \frac{1}{n} \sum_{k=1}^{n} & \exp \left(2 \pi i a_{k} / 5\right) \\
& =\lim \frac{1}{n}\left\{\sum_{k=1}^{n_{1}} \exp \left(2 \pi i b_{k} / 5\right)+\sum_{k=1}^{n_{2}} \exp \left(2 \pi i c_{k} / 5\right)+\sum_{k=1}^{n_{3}} \exp \left(2 \pi i d_{k} / 5\right)\right\} \\
& =\lim \frac{1}{n}\left\{n_{1}+n_{2} \exp \frac{4 \pi i}{5}+n_{3} \exp \frac{6 \pi i}{5}\right\} \\
& =\frac{1}{2 \theta+\alpha}\left\{\alpha+\theta \exp \frac{4 \pi i}{5}+\theta \exp \frac{6 \pi i}{5}\right\} \\
& =(2 \theta+\alpha)^{-1}\left\{\alpha+\theta\left(2 \cos \frac{4 \pi}{5}\right)\right\}=0 .
\end{aligned}
$$

Thus the sequence $A$ has the property $P_{m}$ for all $m \geqq 2$, but it is clear that $A$ is not uniformly distributed since it contains no integers of the form $5 k \pm 1$.

\section{BIBLIOGRAPHY}

1. H. Weyl, Über die Gleichverteilung von Zahlen modulo eins, Math. Ann. vol. 77 (1916) pp. 313-352.

2. I. Niven, Irrational numbers, Mathematical Association of America, Carus Monograph 11, New York, 1956.

3. E. Borel, Les probabilités dénombrables et leurs applications arithmétiques, Rend. Circ. Mat. Palermo vol. 27 (1909) pp. 247-271.

4. T. Nagell, Introduction to number theory, New York, John Wiley, 1951.

5. R. C. Buck, The measure theoretic approach to density, Amer. J. Math. vol. 68 (1946) pp. $560-580$.

6. Th. Skolem, On certain distributions of integers in pairs with given differences, Math. Scand. vol. 5 (1957) pp. 57-68.

7. Th. Bang, On the sequence $[n \alpha], n=1,2, \cdots$, Math. Scand. vol. 5 (1957) pp. 69-76.

UNIVERSITY OF OREGON,

EUGENE, OREGON 